\title{
MÉTODO AGIL DE DESARROLLO DE OBJETOS DE APRENDIZAJE PARA INGENIERIA (MADOA)
}

\section{AGILE METHOD DEVELOPMENT OF LEARNING OBJECT FOR ENGINEERING}

\author{
Huamaní Gloria ${ }^{1}$, Mery Morales ${ }^{2}$, Roberto Eyzaguirre ${ }^{3}$ \\ RESUMEN
}

\begin{abstract}
El objetivo de este artículo es mostrar el diseño de un método ágil para desarrollar objetos de aprendizaje, para Ingeniería aplicado al curso de simulación, por ser un tema afín a cualquier ingeniero, nos ha permitido obtener 7 objetos de aprendizaje, que podrían ser reusados para diferentes cursos y especialidades.
\end{abstract}

Palabras clave.- Método ágil, Objetos de aprendizaje, Ingeniería de software, Simulación.

\begin{abstract}
The aim of this article is to show an agile method for developing learning objects for Engineering applied to the simulation course, being a related topic any engineer, has allowed us to obtain 7 learning objects that could be reused for different courses and specialties.
\end{abstract}

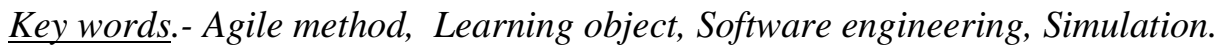

\section{INTRODUCCION}

Las universidades públicas de Lima Metropolitana no ofrecen cursos masivos online abierto. En Open Course Ware de Universia [1] se observa 5 universidades nacionales del país, solo dos a nivel de Lima Metropolitana, los link de UNI y UNMSM no están activos ( visita 02,03 y 04 de enero 2014 /2015). Asimismo, en la Facultad de Ingeniería Industrial y de Sistemas no hay gestión visible de repositorio de contenidos digitales. El centro de información no almacena la producción intelectual de los docentes, tales como: guías, separatas, etc.

Es así como tres docentes nos hemos constituido en un grupo de trabajo para diseñar objetos de aprendizaje del curso de simulación, siendo este un tema útil para todas las especialidades de ingeniería. El Objetivo del trabajo es diseñar un método ágil para desarrollar objetos de aprendizaje para Ingeniería (MADOA). Se ha desarrollado 7 objetos de aprendizaje, para su publicación se ha editado con eXelearning, y el paquete Sharable Content Object Reference Model (SCORM) de Moodle. En ese contexto, el método ágil SCRUM, nos ha servido de referencia para gestionar el proyecto.

MADOA, se describe en tres pasos: Primero se ha desarrollado un prototipo, el mismo ha servido para elaborar un manual de instrucción donde se describe los pasos a seguir y las herramientas a utilizar. En segundo término se mostró el prototipo al especialista temático para la réplica pertinente, cabe mencionar que el reuso es una característica de los objetos de aprendizaje. En tercer término el especialista en tecnología de información hace visible la metadata con el apoyo brindado por dos estudiantes del curso de logística empresarial para la edición y publicación.

\footnotetext{
${ }^{1}$ Doctora y docente investigadora de la Facultad de Ingeniería Industrial y de Sistemas de la Universidad Nacional de Ingeniería, ${ }^{2}$ Doctora y docente investigadora de la Facultad de Ingeniería Industrial y de Sistemas de la Universidad Nacional de Ingeniería, ${ }^{3}$ Doctor y docente investigador de la Facultad de Ingeniería Industrial y de Sistemas de la Universidad Nacional de Ingeniería.
} 
Todos los pasos descritos requieren de un equipo que asume diferentes roles para hacer visible el objeto de aprendizaje, participar en su desarrollo permite compartir información y exteriorizar conocimientos.

Esta publicación se ha estructurado del siguiente modo: en la primera sección, Definición, taxonomía, modelo de diseño, componentes y características de un objeto de aprendizaje (OA); en la segunda sección se muestra aspectos sobre la ingeniería de software como: plataforma tecnológica de código abierto, método ágil SCRUM, en la tercera sección se muestra la metodología - MADOA, los resultados de la investigación, discusión conclusiones y recomendaciones.

\section{OBJETOS DE APRENDIZAJE, TAXONOMÍA, COMPONENTES Y CARACTERÍSTICAS}

Definición.- Un Objeto de Aprendizaje (OA) es un recurso educativo que permite facilitar el proceso de enseñanza aprendizaje La definición de objetos de aprendizaje, y que en su estado digital se convierte en recurso educativo abierto (REA). Sin embargo, según Calzada (2010) [2] la definición de OA ha sufrido una evolución y replanteamientos, existe abundancia de denominaciones de los objetos de aprendizaje, ver Tabla 1.

Tabla 1. Denominación versus responsable de OA.

\begin{tabular}{|c|c|}
\hline Denominación & Responsable \\
\hline Learning resource & ADL \\
\hline $\begin{array}{l}\text { Pedagogical } \\
\text { docuements }\end{array}$ & Ariadne \\
\hline (Reusable & Cisco Systems \\
\hline Information Objetcs) & \\
\hline $\begin{array}{l}\text { RLO (Reusable } \\
\text { Learning Objetcs) }\end{array}$ & $\begin{array}{l}\text { Merlot, Barrit } \\
\text { Alderman (2004) }\end{array}$ \\
\hline $\begin{array}{l}\text { SCO ( Shareable } \\
\text { Content Objects) }\end{array}$ & ADL (SCORM) \\
\hline
\end{tabular}

A su vez, Astudillo [3] cita a (Hodgins, 2000), presenta una definición de Objetos de información (Information object).- $\ll$. Los objetos de información son pequeñas porciones de información en las que se puede dividir el conocimiento, que pueden ser combinadas y utilizadas libremente, como se hace con las piezas de LEGO. Con estos objetos es posible personalizar el aprendizaje utilizando (y reutilizando) el mismo objeto de información $>>$. Por otra parte, para Chan Núñez [4] "un objeto de aprendizaje es una entidad informativa digital que se corresponde (representa) con un objeto real, creada para la generación de conocimientos, habilidades, actitudes y valores, y que cobra sentido en función de las necesidades del sujeto que lo usa". Chang, hace hincapié en que deben ser recursos digitales diseñados con una intencionalidad pedagógica. Además, aunque de manera implícita, ubica a los OA dentro de las corrientes pedagógicas que proponen un aprendizaje centrado en el estudiante.

\section{Taxonomía de objetos de aprendizaje}

El desarrollo de OA inicia desde la ubicación de datos, contenidos en textos, audio, animación, simulación. Si los datos son agrupados. Contextualizados, tienen un significado, entonces se convierten en objetos informativos, mostrados como procedimientos, procesos, resúmenes. Para luego convertirse en objetos de aprendizaje. Para visualizar mejor, se adaptó la taxonomía propuesta por (Hodgings, 2000) citado por Calzada [5]. Ver Figura 2.

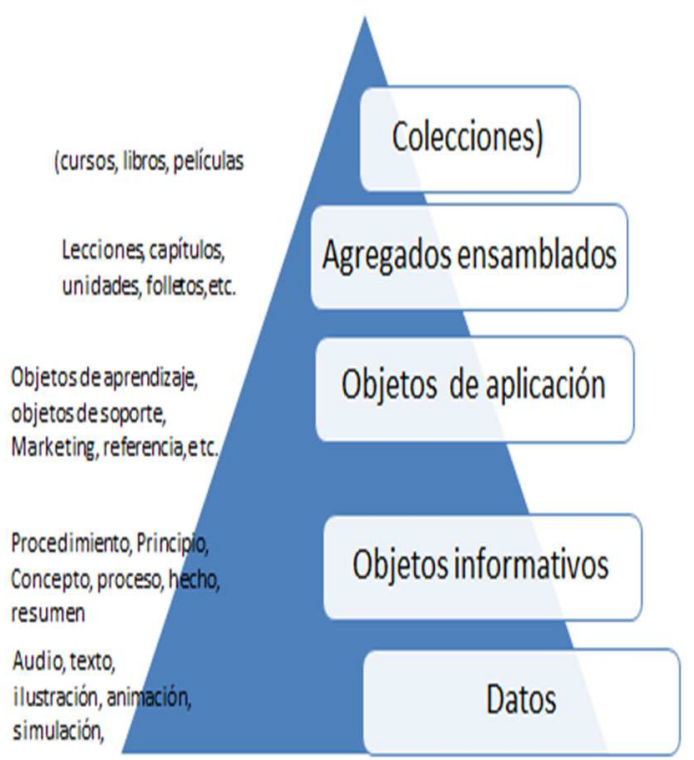

Fig. 2 Taxonomía de objetos de aprendizaje (adaptado de Hodgings). 
Modelo de diseño de un objeto de aprendizaje componentes y/o elementos básicos de los objetos de aprendizaje

El desarrollo de un objeto de aprendizaje se caracteriza por ser un trabajo colaborativo, a su vez, se hace necesaria la identificación de componentes básicos, tales como: aspectos teóricos (contenidos), experiencia práctica, evaluación de los aprendizajes y evaluación. Consideramos muy ilustrativo el modelo de CISCO, citado por Calzada [6]. Ver Figura 3.

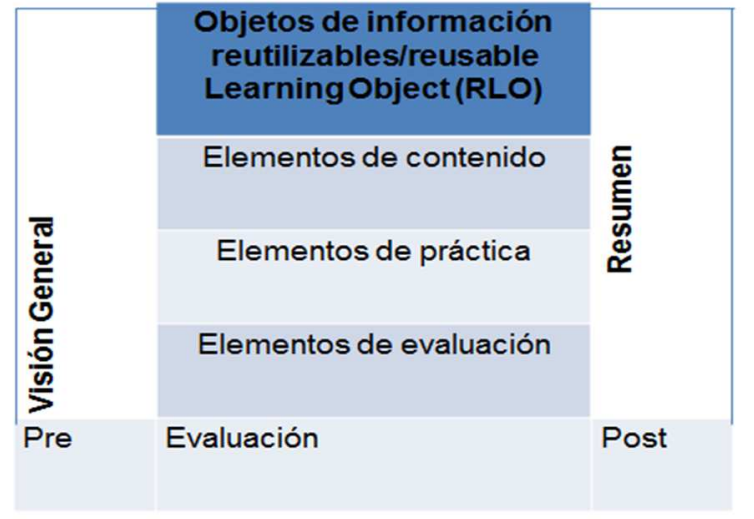

Fig. 3 Modelo de diseño de un objeto de aprendizaje según CISCO.

\section{Características de objetos de aprendizaje}

Diversos autores coinciden acerca de características de los objetos de aprendizaje:

a) Flexibilidad.- El material educativo es usado para usarse en múltiples contextos, debido a su facilidad de actualización, gestión de contenido y búsqueda, esto último gracias al empleo de metadatos.

b) Modularidad.- Posibilidad de entregarlos en módulos, potencia su distribución y recombinación.

c) Reutilización.- El objeto debe tener la capacidad para ser usado en contextos y propósitos educativos diferentes y adaptarse pudiendo combinarse dentro de nuevas secuencias formativas.

d) Granularidad.- Es una concepción de objetos como pequeñas unidades, que pueden ser acopladas y/o adicionadas de diversas maneras, South y Monzón (2000) Citado por Callejas, Hernández y Pinzón. [7] establecen que "la reusabilidad es en gran parte una función del grado de granularidad de los objetos. La reusabilidad del objeto de aprendizaje va a depender en gran medida del grado de granularidad del recurso".

e) Interoperabilidad.- Es la capacidad para poder integrarse en estructuras y sistemas (plataformas) diferentes.

f) Accesibilidad.- Es la facilidad para ser identificados, y recuperados gracias al correspondiente etiquetado a través de diversos descriptores (metadatos) que permitirían la catalogación y almacenamiento en el correspondiente repositorio.

g) Portabilidad.- La mayoría de los sitios web de aprendizaje carece de herramientas convenientes en el empaquetado de la información que los usuarios necesitan; por lo tanto esto dificulta el intercambio de la información publicada en los sitios web, no permiten ser extraídos y reutilizados, así lo afirman Huang, Tsai, Dai [8].

\section{INGENIERIA DE SOFTWARE, METODO AGIL, PLATAFORMA TECNOLOGICA PARA DESARROLLO DE OBJETOS DE APRENDIZAJE}

Las técnicas, métodos, herramientas de Ingeniería de Software han evolucionado. Es en ese contexto que, se aplicó para la gestión de proyecto el método ágil SCRUM, el desarrollo incremental y desarrollo rápido de aplicaciones para desarrollar el prototipo

\section{Antecedentes}

En nuestra búsqueda de métodos de desarrollo de objetos de aprendizaje hemos ubicado: Modelo Instruccional para el diseño de Objetos de Aprendizaje (MIDOA), Metodología para el Desarrollo de Objetos de Aprendizaje (MEDOA), y metodología desarrollada por Universidad del Valle de Colombia. En la Tabla 2 se presenta un análisis comparativo (Huamaní, 2014) [9].

En la universidad del Valle desde la Dirección de Nuevas Tecnologías y Educación Virtual (DINTEV) - Vicerrectoría Académica se hace visible el desarrollo de recursos digitales educativos y en sus boletines se trata diversos temas. 
Siendo un tema de interés: la docencia en entornos virtuales la virtualidad no desplaza al profesor [10] $<$ las profesoras Ocampo, Sanabria y Toro coinciden en que los materiales para la virtualidad tienen que proponer cosas distintas a los textos escritos; se trata de ofrecer al alumno otras posibilidades de acuerdo con su estilo de aprendizaje, propiciando una enseñanza activa y participativa apoyada por diferentes recursos técnicos y audiovisuales $>$. De este modo con el uso de la TIC, el profesor se vuelve mediador, motivador y acompañante del proceso de aprendizaje del estudiante. La propuesta de Universidad del Valle, evoluciona periódicamente. De acuerdo a Borrero, Cruz, et. al. [11] $<<$ se creó una "metodología de diseño de Objetos de
Aprendizaje" la cual es producto de la combinación de un modelo pedagógico y un modelo de ciclo de vida de desarrollo de software. Está constituida por 5 fases a saber: formulación y planificación, análisis, ingeniería, generación de páginas y pruebas, y evaluación del cliente". Han aplicado el modelo incremental, el espiral y el IWEB. Siendo IWEB un modelo de ciclo de vida para el desarrollo de software en el que se han basado para el proceso de desarrollo de software educativo en la Dintev, ya que con él se puede cubrir en su totalidad el ciclo de vida del software a desarrollar, tomando en cuenta los riesgos que se tienen, además, que permite desarrollar versiones cada vez más completas, gracias a su enfoque evolutivo >>.

Tabla 2. Análisis comparativo de métodos de desarrollo de objetos de aprendizaje.

\begin{tabular}{|c|c|c|}
\hline $\begin{array}{l}\text { U Valle Metodología de } \\
\text { diseño de OA }\end{array}$ & $\begin{array}{c}\text { MEDOA Metodología para el } \\
\text { desarrollo de OA }\end{array}$ & $\begin{array}{l}\text { MIDOA Modelo Instruccional } \\
\text { para el diseño de OA }\end{array}$ \\
\hline $\begin{array}{l}\text { 1. Formulación } \\
\text { planificación }\end{array}$ & Planeación & Análisis \\
\hline 2. Análisis & Análisis & Diseño \\
\hline Ingeniería & Diseño & Desarrollo \\
\hline $\begin{array}{l}\text { 4. Generación de } \\
\text { páginas y pruebas }\end{array}$ & Implementación & \\
\hline $\begin{array}{l}\text { 5. } \begin{array}{l}\text { Evaluación del } \\
\text { cliente. }\end{array}\end{array}$ & $\begin{array}{ll}\text { 5. } & \text { Validación } \\
\text { 6. } & \text { Implantación y } \\
\text { 7. } & \text { Mantenimiento }\end{array}$ & $\begin{array}{l}\text { 4valuación a través de } \\
\text { diseño instruccional } \\
\text { proceso basado en } \\
\text { ingeniería de software }\end{array}$ \\
\hline
\end{tabular}

Fuente: Huamaní en TELEDU 2014.

Por lo general corresponde a la etapa de análisis el diseño didáctico, donde se debe tomar decisiones sobre objetivos, contenidos, metodología, evaluación del contenido y aplicación.

Una vez que se ha definido el diseño didáctico se diseña el contenido. Al diseño de contenido corresponde la selección y producción de materiales textuales y no textuales como imagen, video, sonido.

\section{Plataforma tecnológica de código abierto}

La gran mayoría de docentes utiliza aula virtual, en una plataforma que el centro educativo disponga.
En Lima metropolitana las universidades públicas utilizan MOODLE, sigla que corresponde a Modular Object Oriented Dynamic Learning Environment. Moodle sirve para Gestión de sistemas de ambientes de aprendizaje/ course management system o learning management system (CMS/LMS) Basado en PHP y MySQL, Postgre SQL.

Se utiliza MOODLE, entre otras razones por ser de código abierto y por su accesibilidad, así presenta contenidos del curso, enlaza con otros materiales, genera cuestionarios y los aplica, los alumnos envían sus tareas, participan en chats, foros de 
discusión, para proporcionar comentarios sobre el material o el desarrollo del curso.

Sin embargo, unos pocos prestan atención al paquete SCORM, este permite "empacar" una clase utilizando objetos de aprendizaje.

\section{Herramientas - editores de autor}

Existen editores de autor tales como: Reload, eXelearning, Glomaker, Autore, Softchalk. Por su accesibilidad, disponibilidad, y facilidad de uso se ha utilizado eXelearning [12].

\section{Método ágil}

Los métodos ágiles más conocidos, según Casallas [13] son: Extreme Programming (XP), Scrum, Adaptive Software Development (ASD), Crystal Clear y otras metodologías de la familia Crystal, Lean software development, Feature Driven Development, DSDM.

El Scrum es un marco de trabajo de una forma iterativa e incremental. El desarrollo del producto o proyecto, se estructura en ciclos de trabajo llamados Sprints (también conocidos como iteraciones).

Estas iteraciones no deben durar más de cuatro semanas cada una (siendo dos semanas la duración lo habitual) y tienen lugar una tras otra sin pausa entre ellas. Los Sprints tienen un periodo de trabajo, finalizan en una fecha determinada independientemente de si el trabajo ha finalizado por completo o no, y jamás se prorrogan.

Según Ekas [14] $<<$ Los equipos ágiles tienden a ser muy productivos desde la primera iteración hasta su lanzamiento y su ritmo tiene que ser gestionado de modo que no se produzca agotamiento $>$. Un equipo ágil funciona de manera productiva durante todo el ciclo que mantienen este código de trabajo con cada iteración, permiten realizar pruebas de rendimiento y sistemas desde el principio, pudiendo empezar en las primeras iteraciones.

De este modo, defectos críticos como problemas de integración se descubren antes, la calidad general del producto es mayor y el equipo funciona de manera más productiva durante todo el ciclo de desarrollo〉>.

\section{METODOLOGIA - MADOA}

El Desarrollo de MADOA contempla tres fases: Planeación, ejecución y evaluación (Ver Figura 4).

En este proyecto se describe la ejecución.

FASES para obtener MADOA

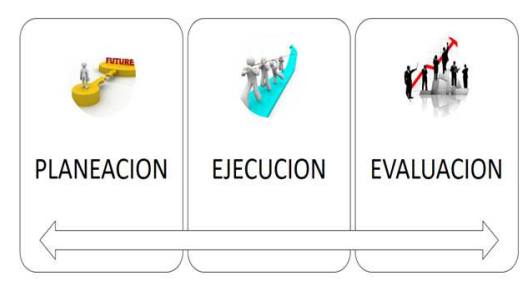

Fig. 4 Fases de MADOA.

En la fase de ejecución del desarrollo de objetos de aprendizaje, se ha observado y replicado el prototipo Objeto de aprendizaje OA mrp. De este modo, se evidencia la conversión de datos en objetos de información, el mismo que sirve para desarrollar un OA.

Para describir y elaborar un video se ha desarrollado un OA de compras. En la selección de textos, videos y material, se hizo una revisión documental. Para el aprendizaje de desarrollar contenidos se aplicó la heurística por un lado y por otro lado el desarrollo incremental para el reuso.

En el desarrollo de MADOA, hay dos tipos de investigación: exploratoria y descriptiva.

La primera etapa es exploratoria, por haber revisado documentos y otros trabajos de investigación.

En una segunda etapa ha sido descriptiva, en esta etapa se ha observado el prototipo OA sobre mrp, por un lado, y por otro se ha constituido un equipo de trabajo que asuma roles de especialista en aspectos técnicos y otro de aspectos pedagógicos, de este modo se evidencia la necesidad de establecer una red de personas que comparten el conocimiento obtenido. El soporte técnico ha sido realizado por dos alumnos de logística, uno de ellos capacitó al otro.

En una tercera etapa, se ha convertido datos en objetos de información. En esta etapa, se ha recurrido a la investigación documental, revisión 
de tópicos sobre simulación, dando lugar a la descripción de la Metadata.

La cuarta etapa es cuasi experimental a esta etapa corresponde la edición y empaquetado de objetos de aprendizaje. Se ha editado contenidos del objeto de aprendizaje, utilizando la herramienta eXelearning, y se ha empaquetado en SCORM MOODLE.

La quinta etapa corresponde a la publicación. Los 7 objetos se han "subido" en la plataforma fiisvirtual.uni.edu.pe, curso simulación del Área de Sistemas y telemática: ST124A.

\section{RESULTADOS}

Método Ágil de Desarrollo de Objetos de Aprendizaje (MADOA)

Para gestionar el desarrollo de objetos de aprendizaje mediante MADOA se aplicó el método ágil SCRUM.

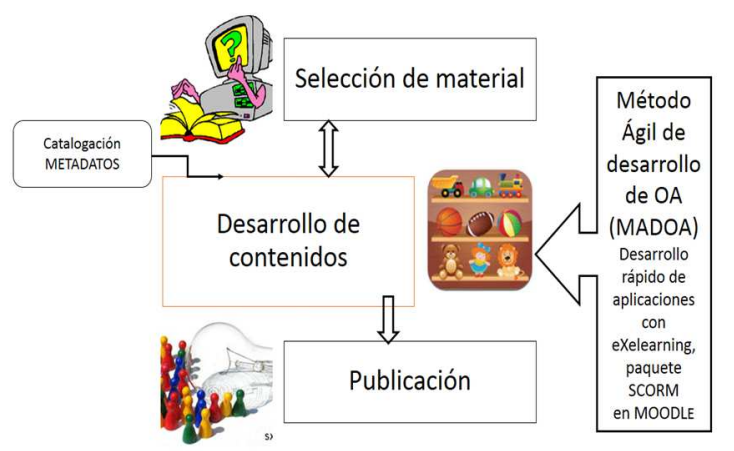

Fig. 5 MADOA v01.1.

Para desarrollar los 7 objetos de aprendizaje para curso de Simulación, el experto en el tema ha seleccionado el material. A partir de ello, se ha desarrollado los contenidos utilizando la herramienta de autor eXelearning. Por otro lado, se ha descrito la metadata en Dublin Core, luego se ha exportado a SCORM para subir a la plataforma MOODLE y finalmente ser publicados y visto por los usuarios.

\section{Metadata}

Cada objeto de aprendizaje requiere una catalogación para ser recuperada. Ver Tablas 3 y 4.
Tabla 3. Información de Metadatos para OA. Tema 2 categoría "5. Uso educativo".

\section{METADATOS INFORMACION}

\section{USO EDUCATIVO}

5.1 Tipo de Expositiva
Interactividad

5.2 Tipo de Recurso Página Web Educativo

5.3 Nivel de Bajo Interactividad

5.4 Densidad Media Semántica

5.5 Destinatarios Estudiantes

5.6 Contexto Educación Superior

5.7 Rango de edad No especificado

5.8 Dificultad Fácil

5.9 Tiempo de $\quad 10$ horas Aprendizaje

5.10 Descripción En "introducción de OA"

5.11 Idioma Español

Tabla 4. Información de Metadatos OA. Tema 2 categoría "6. Derechos".

\begin{tabular}{ll}
\hline \multicolumn{1}{c}{ METADATOS INFORMACION } \\
\hline \multicolumn{1}{c}{ DERECHOS } \\
$\begin{array}{l}\text { 6.1 Agrupa los derechos SI } \\
\text { de propiedad intelectual y } \\
\text { condiciones para el uso } \\
\text { del OAs }\end{array}$ \\
6.2 Coste & NO \\
\hline
\end{tabular}




\section{Publicación y edición de objetos de aprendizaje de curso de simulación}

Se editó un video para mostrar el uso del editor de autor, SCORM y Moodle [15].

La publicación del objeto de aprendizaje se realizó en la plataforma de fiisvirtual.uni.edu.pe [16] según se muestra en la Figura 6.

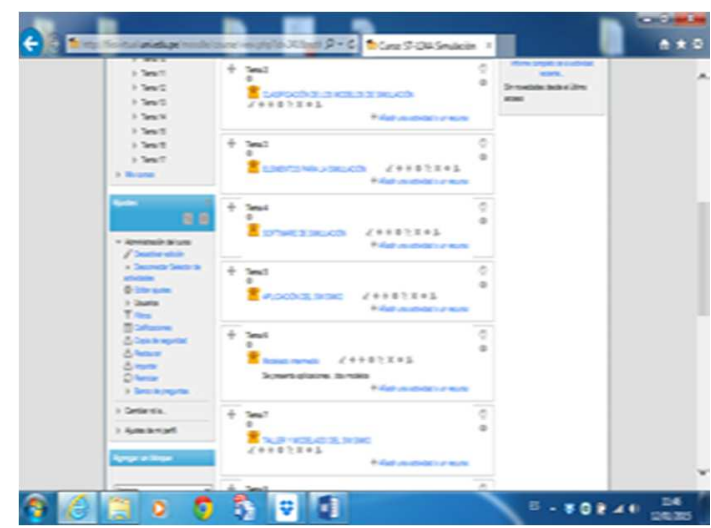

Fig. 6 Objetos de aprendizaje de simulación.

\section{AGRADECIMIENTOS}

Agradecemos a Daniel Moreno, quién ha editado el objeto de aprendizaje mrp a Juan Aldaba por su colaboración en el desarrollo de OA "compras" para facilitar la labor de Joaquín Salcedo, quién editó el manual de diseño instruccional v01.1 en formato video. Al estudiante Michael Larico por apoyar en la edición y publicación de (6/7) objetos de aprendizaje.

\section{CONCLUSIONES}

Se ha consolidado un equipo de trabajo en el desarrollo de objetos de aprendizaje.

Se aplicó método ágil en la ejecución del proyecto facilitando el desarrollo de OA, intercambio de experiencias y aprendizajes.

\section{RECOMENDACIONES}

Para facilitar la visibilidad de la producción intelectual es necesario seguir promoviendo la formación de equipos de trabajo de alto rendimiento, fomentando el uso de editores de autor, elaboración de vídeo tutorial.
Para permitir la reusabilidad e interoperabilidad de objetos de aprendizaje entre docentes, se requiere un repositorio, para almacenar los datos, objetos de información y objetos de aprendizaje.

\section{DISCUSION}

Weiyong Zheng (2013) [17], en su trabajo de investigación se centra en el diseño y la realización de sistemas de gestión de recursos de educación/ Educational Resource managment (ERM), basada en el aprendizaje de metadatos de objetos. El SISTEMA: puede correr en el sistema operativo Windows, y no en Linux; no puede realizar la operación distribuida y adopta la base de datos SQL server.

Por otra parte para Salas, Álvarez et. al. [18], usaron las herramientas Flash, Reload Editor y Reload SCORM Player, para la implementación de los OA.

\section{REFERENCIAS}

1. Universia disponible

en http://ocw.universia.net/es/institucionesintegrantes-iberoamericanasopencourseware.php

2. Calzada, F., "Repositorios, bibliotecas digitales y CRAI". Alfagrama ediciones, Argentina, 2010.

3. Astudillo, G, "Análisis del estado del arte de los objetos de aprendizaje. Revisión de su definición y sus posibilidades". Trabajo final en Maestría: "Informática Aplicada a la Educación" Universidad Nacional de La Plata. Argentina, 2011. http://sedici.unlp.edu.ar/bitstream/handle/109 15/4212/Documento completo.pdf?sequence $=1$

4. Chan Chan, Núñez, "Objetos de aprendizaje", México: Trillas, 2002.

5. Hodgings, "Taxonomía de objetos de aprendizaje en Calzada (Op. Cit)", pp. 76.

6. CISCO. "Modelo de diseño de Objetos de aprendizaje en Calzada (Op. Cit)", pp. 90.

7. Callejas, M., Hernández, E., Pinzón, J., "Objetos de aprendizaje un estado del arte" Entramado. Unilibre Cali Vol.7 No. 1, 2011 (Enero - Junio) pp. 176-189. Disponible en http://www.scielo.org.co/pdf/entra/v7n1/v7n1 a12.pdf 
8. Huang, Tsai, Dai, "Building Learning Object with Information Object Model", IEEE International Conference on Teaching, Asessment and Learning for Engineering. 2629 Agosto, 2013 IEEE. Disponible en http://www.ieee.org

9. Huamaní, G., "Gestión de conocimiento para desarrollar objetos de aprendizaje para logística empresarial", TELEDU 2014 Medellín Colombia en http://www.portafolioconsultores.org/memori asteledu2014/

10. Universidad del Valle, "La docencia en entornos virtuales la virtualidad no desplaza al profesor", Santiago de Cali, Octubre de 2013 - Año 10 No. 29. Boletín informativo dela DINTEV en

http://dintev.univalle.edu.co/saberabierto/adju ntos/saberabierto_ed29.pdf

11. Borrero, M., Cruz E., Mayorga, S., Ramírez, "Una metodología para el diseño de Objetos de Aprendizaje", La experiencia de la Dirección de Nuevas Tecnologías y Educación Virtual, Dintev, de la Universidad del Valle. Universidad del Valle, 2009.

12. http://exelearning.net

13. Casallas Casallas, R., "Metodologías ágiles de desarrollo de software", en http://www.academia.edu/870447/Metodolog \%C3\%ADas_\%C3\%A1giles_de_desarrollo_d e_Software
14. Ekas, L., Disponible 12.01 .15 en https://www.ibm.com/developerworks/comm unity/blogs/rationalspain/entry/5_beneficios_ de_las_metodolog_C3_ADas_C3_A1giles_e n_el_desarrollo_de_sistemas_de_software5?1 ang=en

15. Huamaní, G., Salcedo, J., "Manual de diseño instruccional para desarrollo de objetos de aprendizaje", Lima, UNI FIIS 31.12.14. https://www.youtube.com/watch?v=LIU_ifOu Xho

16. "Objetos de aprendizaje de curso de simulación",

http://fiisvirtual.uni.edu.pe/moodle/

17. Weiyong, Zheng, "Design and Realization of Educational Resources Resources Management System Based on Learning Object", Metadata. Seventh International Conference. 2013 IEEE. Disponible en http:// www.ieee.org

18. Salas Alvarez, et.al. Bula Herazo, Peña Salgado., "En su trabajo Diseño e implementación de Objetos de Aprendizaje basado en Tecnologías estándares para soportar e-learning", Revista Cognición Noviembre 2007- Año 3 - No. 11, pp. 66.

Correspondencia: therehuamani@gmail.com

Recepción de originales: octubre 2014

Aceptación de originales: diciembre 2014 